\title{
Correction to: Effect of 650-nm low-level laser irradiation on c-Jun, c-Fos, ICAM-1, and CCL2 expression in experimental periodontitis
}

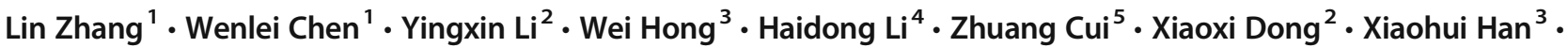 Gang Bao ${ }^{1} \cdot$ Li Xiao $^{1} \cdot$ Pengfei Gao ${ }^{1}$. Yonglan Wang ${ }^{1}$}

Published online: 10 December 2019

(C) Springer-Verlag London Ltd., part of Springer Nature 2019

\section{Correction to: Lasers in Medical Science https://doi.org/10.1007/s10103-018-2662-y}

After publication of our article [1] we realized that we had not acknowledged that some of the text overlaps with a previous publication [2]. We apologize to readers for this error.

1. Lin Zhang, Wenlei Chen, Yingxin Li, Wei Hong, Haidong Li, Zhuang Cui, Xiaoxi Dong,Xiaohui Han, Gang Bao, Li Xiao, Pengfei Gao, Yonglan Wang. Effect of 650-nm lowlevel laser irradiation on c-Jun, c-Fos, ICAM-1, and CCL2 expression in experimental periodontitis. Lasers Med Sci (2018). https://doi.org/10.1007/s10103-018-2662-y

The online version of the original article can be found at https://doi.org/ 10.1007/s10103-018-2662-y

Yonglan Wang

enhai813@sina.com

1 School of Dentistry, Hospital of Stomatology, Tianjin Medical University, 12 Qi Xiang Tai Street, Heping District, Tianjin 300070, China

2 Institute of Biomedical Engineering, Academy of Medical Science and Peking Union Medical College, Tianjin, China

3 Department of Histology and Embryology, School of Basic Medical Sciences, Tianjin Medical University, Tianjin, China

4 Department of Biochemistry and Molecular Biology, School of Basic Medical Sciences, Tianjin Medical University, Tianjin, China

5 School of Public Health, Tianjin Medical University, Tianjin, China
2. Zhang L, Zhao J, Kuboyama N, Abiko Y. Low-level laser irradiation treatment reduces CCL2 expression in rat rheumatoid synovia via a chemokine signalling pathway. Lasers Med Sci. 2011 Sep;26(5):707-17. doi: 10.1007/ s10103-011-0917-y. Epub 2011 May 4. PubMed PMID: 21541773.

Publisher's note Springer Nature remains neutral with regard to jurisdictional claims in published maps and institutional affiliations. 